\title{
THE SCATTERING OF LIGHT BY A LARGE CONDUCTING SPHERE
}

(Second Paper.)

By J. W. Nicholson.

[Received April 29th, 1911. - Read May 11th, 1911. -

Revised November 29th, 1911.]

IN the first paper on this subject it was shewn that the disturbance radiated from a large perfectly conducting sphere on which plane waves are incident, when calculated from the harmonic series to which the electromagnetic theory leads, is in accord, to a first approximation, with the result of a calculation by the ordinary method of optics.

This conclusion was shewn to be valid when the point, at which the effect is determined, does not lie close to the axis, defined as the diameter of the sphere drawn parallel to the direction of propagation of the incident waves.

In this paper, points near the axis are examined, and they are found to present no exception to the statement.

As it is necessary to make frequent reference to formulæ in the first paper, such formulæ will be denoted by a square bracket [ ]. The notation is the same as before.

Corresponding to a system of plane waves travelling along the direction $x$, and incident on the positive side of the sphere, containing an electric polarisation $\left(f_{0}, g_{0}, h_{0}\right)$ and magnetic force $\left(\alpha_{0}, \beta_{0}, \gamma_{0}\right)$ given by

$$
\left.\begin{array}{l}
\left(f_{0}, g_{0}, h_{0}\right)=\left(0,0, e^{\imath k x}\right) \\
\left(\alpha_{0}, \beta_{0}, \gamma_{0}\right)=-4 \pi V\left(0, e^{\imath k x}, 0\right)
\end{array}\right\},
$$


it was shewn in [16] that the polarisation in the scattered waves is

$$
\begin{aligned}
(f, g, h)= & -\frac{(x, y, z)}{k^{2} r^{3}} e^{-\imath k r} \Sigma_{1}^{\infty} 2 m \cos \left(\phi_{n}+\chi_{n}\right) \sin \theta \sin \phi \frac{d P_{n}}{d \mu} e^{\iota \xi} \\
& +i e^{-\imath k r} \sum_{1}^{\infty} \frac{2 m}{k n, n+1} \cos \left(\phi_{n}+\chi_{n}\right) \frac{\partial}{\partial(x, y, z)} \sin \theta \sin \phi \frac{d P_{n}}{d \mu} e^{\iota \xi} \\
& -e^{-\imath k r} \sum_{1}^{\infty} \frac{2 m}{k r n, n+1} \sin \phi_{n}\left(y \frac{\partial}{\partial z}-z \frac{\partial}{\partial y}, \ldots, \ldots\right) \\
= & S_{1}+S_{2}+S_{3} \text { (say), } \\
& \times \sin \theta \cos \phi \frac{d P_{n}}{d \mu} e^{\iota \xi-\imath x_{n}}
\end{aligned}
$$

where

$$
m=n+\frac{1}{2}, \quad \xi=\phi_{n}+\chi_{n}+n \pi .
$$

The differentiations may be taken outside the summations on the understanding that they act only on $\theta$ and $\phi$, and not on $r$. The angles $\theta$ and $\phi$ are given by

$$
x=r \cos \theta, \quad y=r \sin \theta \cos \phi, \quad z=r \sin \theta \sin \phi .
$$

In order to include points near the axis, we may employ, instead of the asymptotic expansion of $P_{n}(\mu)$, the exact Mehler-Dirichlet formula,

$$
P_{n}(\mu)=\frac{2}{\pi} \int_{0}^{\theta} \frac{\cos m \omega d \omega}{\sqrt{2} \sqrt{ }(\cos \omega-\cos \theta)},
$$

which therefore takes the place of

$$
(2 / m \pi \sin \theta)^{\frac{1}{2}} \cos \left(m \theta-\frac{1}{4} \pi\right) \text {. }
$$

The value of $v$ for a series of oscillatory type

$$
\Sigma u e^{s v v} \text {, }
$$

$(z=k a)$, thus contains $\omega$ in place of $\theta$.

From the differential equation satisfied by the zonal harmonic,

$$
\sin ^{2} \theta \frac{d P_{n}}{d \mu}=-n . n+1 \int_{1}^{\mu} P_{n} d \mu
$$

and by a recurrence formula,

$$
2 n+1 \int_{1}^{\mu} P_{n} d \mu=\int_{1}^{\mu}\left(\frac{d P_{n+1}}{d \mu}-\frac{d P_{n-1}}{d \mu}\right) d \mu=P_{n+1}-P_{n-1} .
$$

Hence

$$
\begin{aligned}
\frac{2 n+1}{n . n+1} \sin ^{2} \theta \frac{d P_{n}}{d \mu}=P_{n-1}-P_{n+1} & =\frac{2}{\pi} \int_{0}^{\theta} \frac{\cos (m-1) \omega-\cos (n+1) \omega}{\sqrt{ } 2 \sqrt{ }(\cos \omega-\cos \theta)} d \omega \\
& =\frac{4}{\pi} \int_{0}^{\theta} \frac{\sin \omega \sin m \omega d \omega}{\sqrt{ } 2 \sqrt{ }(\cos \omega-\cos \theta)} .
\end{aligned}
$$


Let $U$ denote an operation which, acting on $F$, has the effect $U . F \equiv \frac{4 e^{-i k r}}{\pi}\left(-\frac{a}{k^{2} r^{3}}+\frac{\iota}{k n, n+1} \frac{\partial}{\partial a}\right) \frac{n \cdot n+1 \sin \phi}{\sin \theta} \int_{0}^{\theta} \frac{\sin \omega \cdot F d \omega}{\sqrt{2 \sqrt{(\cos \omega-\cos \theta)}}}$,

where $a$ denotes $(x, y, z)$ as in the first paper. Then, by (2),

$$
S_{1}+S_{2}=\Sigma_{n=1}^{\infty} U \cos \left(\phi_{n}+\chi_{n}\right) \sin m \omega \cdot e^{\imath \phi_{n}+i \chi_{n}+\imath \imath \pi},
$$

where no factor of the operation $U$ is oscillatory. We shewed before that $\chi_{n}$ does not oscillate in the neighbourhood of possible zero points, which are determined from [22]. In fact, it is of order $z^{-1}$ there, where $z=k a$. A comparison with [22-27] shews that such points, when they occur, are given by $t=\sin \frac{1}{2} \omega$, where $t$ denotes $m / z$. For the oscillatory portions of the exponents only differ from their old values by a substitution of $\omega$ for $\theta$.

Moreover, these exponents being

$$
\left.\begin{array}{l}
z\left(v_{1}, v_{2}\right)=2 \phi_{n}+m \pi \pm m \omega \\
z\left(v_{3}, v_{4}\right)=m \pi \pm m \omega
\end{array}\right\}
$$

it follows, as in [27], that only $z v_{2}$ leads to a point of this nature, when $\omega$ is not zero or $\pi$. If $\omega$ is zero, $z v_{1}$ also leads to such a point $t=0$. We shall return to this case later. It arises when $m \theta$ is very small, or $z \theta$ small. If $t=\left(n+\frac{1}{2}\right) / z$,

$$
\phi_{n}=z\left\{\left(1-t^{2}\right)^{3}+t \sin ^{-1} t-\frac{1}{2} \pi t\right\}+\frac{1}{4} \pi,
$$

gives the value of $\phi_{n}$ near the point $t=\sin \frac{1}{2} \omega$. It would cesse to do so if $\omega$ were nearly $\pi$. This case does not arise in the present paper. The complete value of $S_{1}+S_{2}$ is

$$
S_{1}+S_{2}=-\frac{1}{4} \Sigma_{1}^{\infty} U\left\{e^{i v_{1}+2 x_{n}}-e^{i v_{2}+2 x_{n}}+e^{i v_{3}}-e^{i v_{4}}\right\},
$$

and rejecting the three series without a zero point, we may write, approximately,

$$
S_{1}+S_{2}=\frac{1}{4} \Sigma_{1}^{\infty} U \cdot e^{i v_{2}+2 \times x_{n}} .
$$

If the suffix refers to the zero point henceforth, $v_{9}$ being now $v$ in (12), then, by [26], the leading term in the sum is

$$
S_{1}+S_{2}=\frac{1}{2} U_{0}\left(\frac{\pi z}{2 v_{0}^{\prime \prime}}\right)^{\frac{3}{2}} e^{u v_{0}+2 x_{0}+z i m},
$$

the accent denoting $\partial / \partial t$ or $z \partial / \partial m$, and $v_{0}^{\prime \prime}$ being positive. 
In this formula, with $\chi_{0}=0$,

$$
\begin{aligned}
z v=z v_{2} & =2 \phi_{n}+m \pi-m \omega \\
& =2 z\left\{\left(1-t^{2}\right)^{2}+t \sin ^{-1} t\right\}-z \omega t+\frac{1}{2} \pi,
\end{aligned}
$$

so that, when $t=\sin \frac{1}{2} \omega$,

$$
\begin{gathered}
z v_{0}=2 z \cos \frac{1}{2} \omega+\frac{1}{2} \pi, \quad v_{0}^{\prime}=0, \\
v_{0}^{\prime \prime}=2\left(1-t^{2}\right)^{-\frac{1}{2}}=2 \sec \frac{1}{2} \omega,
\end{gathered}
$$

and is positive, so that

$$
S_{1}+S_{2}=\frac{1}{4} i U_{0}\left(\pi z \cos \frac{1}{2} \omega\right)^{\frac{1}{3}} e^{2 i z \cos \xi \omega+1 i \pi} .
$$

Since $n . n+1=m^{2}-\frac{1}{4}$, the value of this product at the zero point $m=z \sin \frac{1}{2} \omega$ is effectively $z^{2} \sin ^{2} \frac{1}{2} \omega$, so that

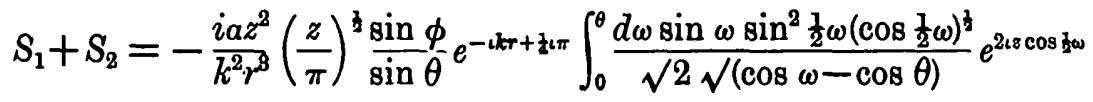

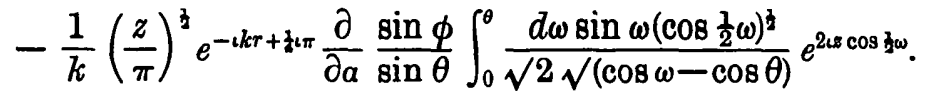

The main term of each integral arises from the upper limit if $\theta$ is not too small. Writing $\cos \omega=\cos \theta+\lambda^{2}$,

$$
\begin{aligned}
I_{1} & \equiv \int_{0}^{\theta} \frac{d \omega \sin \omega \sin ^{2} \frac{1}{2} \omega\left(\cos \frac{1}{2} \omega\right)^{\frac{1}{2}}}{\sqrt{2} \sqrt{(\cos \omega-\cos \theta)}} e^{2 \omega \cos \frac{\jmath}{\omega} \omega} \\
& =\sqrt{ } 2 \sin ^{2} \frac{1}{2} \theta\left(\cos \frac{1}{2} \theta\right)^{\frac{1}{2}} \int_{0}^{2^{2} \sin \frac{3}{3} \theta} d \lambda e^{L v\left[2\left(1+\cos \theta+\lambda^{2}\right)\right]},
\end{aligned}
$$

with an error of relative order $z^{-1}$ by the usual theory of such integrals. To the same order,

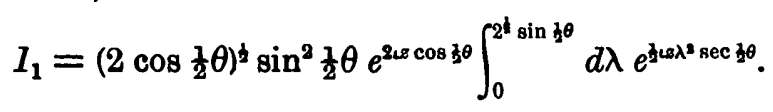

The error involved in supposing that the integral extends to infinity is $(z \theta)^{-1}$ relatively to the final result. To this order,

on reduction.

$$
I_{1}=\frac{1}{2}\left(\frac{\pi}{z}\right)^{3} \sin \theta \sin \frac{1}{2} \theta e^{2 \pi s \cos 3 \theta+3 ! \pi},
$$

The first term of $S_{1}+S_{2}$ thus becomes, in (15),

$$
\frac{\alpha a^{2}}{2 r^{3}} \sin \phi \sin \frac{1}{2} \theta e^{-\imath k r+2 \imath k a \cos 3 \theta} \text {. }
$$


To the same order $(z \theta)^{-1}$, the second term becomes, in a similar manner,

$$
-\frac{\iota}{k} \frac{\partial}{\partial \alpha} \frac{\sin \phi \cos \frac{1}{2} \theta}{\sin \theta} e^{-2 \mathrm{k} k a \cos \frac{1}{2} \theta} \text {. }
$$

By addition, we obtain the formula [31], which may be developed as before. The sum $S_{1}+S_{2}$ has therefore been correctly estimated, subject to a relative error $(z \theta)^{-1}$, the maximum error in the present investigation. A similar detailed treatment may be given to $S_{8}$, with the same result. Its reproduction here does not seem necessary.

We may conclude, therefore, that the extent to which the formula of the first paper have been shewn to be valid near the positive side of the axis is determined by the relative smallness of $(z \theta)^{-1}$ compared with unity. Thus for the numerical case of visible light and a sphere of an inch diameter, the error would not exceed one per cent. if $(z \theta)^{-1}=10^{-2}$, or as $z=1 \cdot 410^{6}$, if $\theta$ were of order $10^{-4}$, so that $\theta^{2}$ is itself negligible to the same order.

We shall see that the error is actually of relative order $z^{-1}$.

With this conclusion, we may adopt a simpler method of investigating points so close to the axis, in order to discover whether the nature of the effect changes at all. It cannot be expected to do so.

When $z \theta$ becomes small, another series neglected above, because it had no zero point, becomes equally important. This is the series whose exponent contains

$$
2 \phi_{n}+m \pi+m \omega,
$$

where $\omega$ is never greater than $\theta$, for if $\theta=0$, this series also has a zero point $t=0$.

Therefore we cannot obtain a correct result by proceeding to the limit, when $\theta=0$, of the Fresnel integral in the last investigation, and instead of doing this, and subsequently adding the other series, it is simpler to find the effect on the axis as follows.

We may ignore the series $S_{1}$ at once, for it is proportional to $1 / r^{2}$. This was ultimately ignored before.

If $\alpha$ denotes the Cartesian coordinates $(x, y, z)$ in turn,

$$
\partial / \partial \alpha\left(\sin \theta \sin \phi d P_{n} / d \mu\right)
$$

may be calculated by differentiating $\sin \theta \sin \phi$ only, if $\theta$ is small, and it is readily shewn that

$$
\left.\begin{array}{l}
\partial \theta / \partial \alpha=(-\sin \theta, \cos \theta \cos \phi, \cos \theta \sin \phi) / r \\
\partial \phi / \partial \alpha=(0,-\sin \phi, \cos \phi) / r \sin \theta
\end{array}\right\} .
$$


Thus $\quad \partial / \partial a(\sin \theta \sin \phi)=\sin \theta \cos \phi \partial \phi / \partial \alpha+\cos \theta \sin \phi \partial \theta / \partial \alpha$

$$
=(0,0,1) / r \text {, }
$$

without approximation on the axis. Therefore, when $\theta=0$, since $d P_{n} / d \mu$ may be replaced, to order $\theta^{2}$, by $\frac{1}{2} n . n+1$, we find

$$
\partial / \partial \alpha\left(\sin \theta \sin \phi d P_{n} / d \mu\right)=n(n+1)(0,0,1) / 2 r,
$$

and in a similar manner,

$$
(y \partial / \partial z-z \partial / \partial y, \ldots, \ldots)\left(\sin \theta \cos \phi d P_{n} / d \mu\right)=n(n+1)(0,0,1) / 2 ;
$$

and therefore, with (2),

or

$$
\begin{aligned}
&(f, g, h)= \frac{e^{-i k r}}{k r}(0,0,1) \Sigma_{1}^{\infty} m \cos \left(\phi_{n}+\chi_{n}\right) e^{i \phi_{n}+i x_{n}+i m \pi} \\
&+\frac{e^{-i k r}}{k r}(0,0,1) \Sigma_{1}^{\infty} i m \sin \phi_{n} e^{i \phi_{n}+i m \pi} \\
&= \frac{e^{-\imath k r}}{2 k r}(0,0,1) \Sigma_{1}^{\infty} m\left(1+e^{\left.2 i \chi_{n}\right)} e^{2 i \phi_{n}+\imath m \pi}\right. \\
& f=0, \quad g=0, \quad h=\frac{e^{-\imath k r}}{2 k r} \Sigma_{1}^{\infty} m\left(1+e^{\left.2 i x_{n}\right)} e^{2 i \phi_{n}+\imath m \pi}\right.
\end{aligned}
$$

This formula for points on the axis is exact to the order $1 / r$.

The direction of vibration in the scattered light on the axis is therefore the same as in the incident light. This would be expected from the symmetry.

The series (23) may be shewn to be absolutely convergent, for let us consider the terms near infinity. In such terms, where $m$ greatly exceeds $z$, we may write*

$$
\tan \phi_{n}=e^{2 t_{n}}, \quad t_{n}=-\frac{1}{2} \log 2+z(\sinh \beta-\beta \cosh \beta), \quad \beta=m / z,
$$

where $t_{n}$ is large and negative. Moreover,

$$
\begin{gathered}
\partial R_{n} / \partial z=-e^{-2 t_{n},} \\
\tan \chi_{n}=-\frac{1}{2} \partial R_{n} / \partial z=\frac{1}{2} e^{2 t_{n}},
\end{gathered}
$$

and tends to infinity. Thus $\chi_{n}$ tends to $\frac{1}{2} \pi$, and $1+e^{2 x_{n}}$ to zero. Effectivels,

$$
\tan \chi_{n}=\frac{1}{4} e^{2 x \beta \cosh \beta}=\tan \left(\frac{\pi}{2}-u\right) \text {, }
$$

\footnotetext{
* Phil. Mag., April, 1910, p. 525.
} 
where

$$
u=4 e^{-2 z \beta \cosh \beta},
$$

so that ultimately

$$
1+e^{2 \imath x_{n}}=8 \iota e^{-2 z \beta \cosh \beta} \text {. }
$$

Thus the modulus series of (23) is ultimately of the form

$$
\frac{4}{k r} \sum m e^{-m \cosh m ; z}
$$

and is absolutely convergent.

Writing

$$
a_{m}=\frac{m}{2 k r}\left(1+e^{\left.2 \imath x_{n}\right)} e^{2 \imath \phi_{n}+\iota m \pi-\imath k r}\right.
$$

then

$$
h=\Sigma_{1}^{\infty} a_{m} .
$$

Consider now the series

$$
h^{\prime}=\Sigma_{1}^{o s} a_{m} e^{-m^{2} \xi i z},
$$

where the index of the exponential contains a new positive parameter $\xi$. Then since the series $h$ is convergent, the generalised Abel-theorem* shews that as $\xi$ tends to zero, $h^{\prime}$ tends to the limit $h$.

Now the zero point in the series $h$ or $h^{\prime}$ is at $t=0$, in whose neighbourhood $\chi_{n}$ may be neglected, and $1+e^{2 \imath \chi_{n}}=2$. With $m=z t=k a t$, then near $t=0$,

$$
\begin{aligned}
2 \phi_{n}+m_{0} \pi & =2 z\left\{\left(1-t^{2}\right)^{\frac{1}{3}}+t \sin ^{-1} t\right\}+\frac{1}{2} \pi \\
& =2 z+\frac{1}{2} \pi+z t^{2},
\end{aligned}
$$

and replacing the summation $h^{\prime}$ by the first approximation to its equivalent integral, $\dagger$

$$
h^{\prime}=\frac{\iota \alpha}{r} e^{2 \imath z-\imath k r} \int_{0}^{\infty} z t e^{-(\xi-\iota) 2 t^{2}} d t \quad(\xi>0) .
$$

The value of the integral is $\frac{1}{2}(\xi-\imath)$, so that

$$
h^{\prime}=\frac{\iota a}{2 r(\xi-\imath)} e^{2 \imath z-\iota \iota r},
$$

whose limit when $\xi=0$ becomes

$$
h=-\frac{a}{2 r} e^{-\imath k r+2 \imath k a},
$$

- Vide, e.g., Bromwich, Theory of Infinite Series, p. 113.

+ Messenger of Mathematics, Oot., 1907. The corresponding integral for $h$ is of a divergent type, and its consideration presents some difficulties. The use of the Abel-theorem which provides the simplest means of avoiding them, was suggested to me by Dr. Bromwich. 
284 The scattering of Light by a large conducting sphere.

which is therefore the value of $h$. This is identical with the limiting value of [40] when $\theta$ is small. Accordingly, [40] is correct for points actually on the axis.

This investigation throws an interesting light on the relative importance of various parts of a harmonic series in problems of this nature. Near the axis, another part of the series becomes important, and is exactly balanced by the change in the former part, so that the result is unaltered. The type of integral in (29) is also different, but follows naturally by the general method of the analysis.

We note that the series without zero points have disappeared together on the axis, where the zero points of series which possess them are at $t=0$. 